\title{
Fate of coronary collateral circulation after aorto-coronary saphenous vein bypass grafts
}

\author{
V. Di Luzio, ${ }^{1}$ P. R. Roy, ${ }^{2}$ E. Sowton, and J. Dow \\ From the Cardiac Department and Surgical X-Ray Theatres, Guy's Hospital, London
}

The pre-and postoperative patterns of coronary artery collateral circulation have been studied in 34 patients who had saphenous vein bypass grafting. When the graft remained patent homocoronary collaterals could not be visualized after operation, but new intercoronary anastomoses frequently developed to other diseased arteries. When the graft and the bypassed artery were both obstructed there was a high incidence (5 out of II) of myocardial infarction despite good preoperative collaterals.

Selective coronary arteriography with modern radiological apparatus allows vessels of $100 \mu \mathrm{m}$ in diameter to be well visualized (Gensini and $\mathrm{Da}$ Costa, 1969), so that coronary collateral vessels can be accurately delineated in man during life (Gensini and $\mathrm{Da}$ Costa, 1969; Sheldon, 1969; Helfant, Kemp, and Gorlin, 1970). The fate of the coronary collateral circulation after myocardial revascularization both in successful and unsuccessful surgery is not well documented. This study has been carried out with the purpose of examining the behaviour of the coronary collateral circulation after aortocoronary saphenous vein bypass grafts in a group of patients who underwent routine postoperative study.

\section{Subjects and methods}

There were 34 patients in the series of whom 28 were men and 6 were women. All patients had coronary saphenous vein bypass surgery for symptoms of severe ischaemic heart disease which were unresponsive to medical therapy. The site of the graft origin from the aorta was marked at surgery with a small opaque marker, as an aid to restudy.

Coronary arteriography was performed before operation and repeated between 2 and 14 months after operation together with saphenous vein graft angiography. In 8 patients at the postoperative study, the venous graft could not be selectively entered despite knowledge of its origin. An aortogram was performed which showed no evidence of graft patency, and in these patients the graft was considered to be occluded. Graft occlusion was con-

Received 5 July 1974 .

${ }^{1}$ British Council Scholarship, October 1972 - August 1973. Present address: Ospedale Cardiologico, Ancona, Italy.

${ }^{2}$ Fellowship supported by the Postgraduate Committee in Medicine, University of Sydney. Present address: National Heart Hospital, Westmoreland Street, London WIM 8BA. firmed at reoperation in 2 of these patients who were symptomatic and in 3 patients the aortogram showed clearly the stumps of the occluded grafts. The coronary artery injections were made either by the Sones or the Judkins technique using Hypaque 65 per cent. The right coronary artery was visualized at $20^{\circ}$ and $40^{\circ}$ in the left anterior oblique position and at $30^{\circ}$ in the right anterior oblique position; the left coronary artery tree at $20^{\circ}, 40^{\circ}$, and $60^{\circ}$ in the left anterior oblique and at $30^{\circ}$ in the right anterior oblique.

The radiographic equipment was a Philips unit which included a rotating couch, a 6-inch $(15.24 \mathrm{~cm})$ image intensifier, and an Eclair $\mathrm{CV}_{35}$ Cameflex camera operating with a speed ranging from 24 to 120 frames/second. Kodak XX negative films were used and they were commercially developed and printed. The images were magnified to approximately $2 \frac{1}{2}$ times the natural size and the films were analysed on a Tage Arno projector.

According to a radiographic classification (Gensini and Esente, 1972) the coronary collateral circulation was divided into 3 types: homocoronary, intercoronary, and mixed. A homocoronary collateral circulation was considered to be present when, together with proximal occlusive disease, the distal filling of one or more branches of the injected coronary artery could be seen to occur through visible anastomotic pathways.

Filling of one or more segments of a diseased coronary tree distal to an occlusive lesion from selective injection of the contralateral coronary artery was accepted as evidence of an intercoronary collateral circulation. In the vast majority of these cases, clear anatomical connexions between the distal ramifications of the left and right coronary artery were seen.

A mixed coronary collateral circulation was considered to be present when, together with a homocoronary functioning anastomotic pathway, an intercoronary collateral circulation was established from one of these anastomotic pathways, i.e. a mixed coronary collateral circulation was not the simple association of a homo- 
coronary with an intercoronary collateral circulation, but the association of a homocoronary with an intercoronary collateral circulation having a common source of blood supply (i.e. left anterior descending anastomosing with obtuse marginal and right posterior descending).

\section{Results}

\section{Preoperative studies}

At the preoperative coronary angiogram all patients showed severe coronary occlusive disease resulting in more than 80 per cent reduction in lumen diameter or a complete obstruction of a main coronary trunk or at least one major branch. The left anterior descending coronary artery was involved in 28 cases, the left circumflex in 4 cases, the main trunk of the left coronary artery in 4 cases, and the right coronary artery in 29 cases. Sixty-eight per cent of patients also showed occlusive lesions of various degree in other minor branches. Collateral pathways could be seen in all patients before surgery. In 80 per cent of the occasions in which an intercoronary collateral circulation was present, clear anatomical connexions between the distal ramifications of the left and right coronary arteries were well delineated. In the remaining cases their existence was deduced by the delayed filling of a coronary branch following the injection of contrast medium into the heterolateral coronary artery.

In 6 patients in whom homocoronary collateral circulations were present no direct continuation was demonstrable between the recipient and the donor artery. Their existence was, however, deduced by the retrograde filling of the obstructed artery from the territory of distribution of the normal adjacent artery.

\section{Postoperative studies}

Patent grafts Forty-four coronary arteries received patent grafts and filled from selective injection of the grafts. In 21 cases there had been some anterograde filling preoperatively shown by injection of the main stem. In 37 cases preoperative filling was seen via collateral vessels. Postoperatively, filling via collaterals was seen in only 3 cases. Thus, establishment of good bypass graft flow was followed by non-visualization of collaterals in 34 cases $(92 \%)$. In no case were new collaterals, not present before operation, visualized.

Occluded grafts Twenty-one arteries received grafts which were classified as occluded at the postoperative study. In 5 the bypassed artery showed some anterograde flow after injection of the main stem, both before and after surgery. Of these 5,4 had demonstrable collaterals before operation, and all 5 had them at the postoperative study. In 4 of these 5, therefore, the insertion of a graft which occluded made little, if any, difference to the collateral circulation. In the remaining 16 there was no anterograde flow through the stenosis postoperatively, though this had been demonstrated in II preoperatively. Twelve of the 16 had collateral filling before bypass grafting, and 15 afterwards.

Overall, 16 of these 21 arteries showed some anterograde flow through the stenosis before operation, but in II $(69 \%$ of the I6) the arterial stenosis had blocked completely by the time of the postoperative study. Despite good collaterals 5 patients developed myocardial infarction in relation to surgery. This suggests that the pre-existing collateral filling was inadequate to maintain perfusion and new collaterals could not develop quickly enough to prevent muscle death.

\section{Types of collateral flow}

When the bypass graft was patent the collateral circulation could not usually be identified postoperatively, and this situation applied whether the existing pattern was homocoronary or intercoronary. In 16 patients with 2 patent grafts collateral circulation to 32 arteries was shown preoperatively. The effects of surgery are shown in the Table. In addition 5 new collateral pathways from the bypassed arteries to other diseased (non-bypassed) vessels were demonstrated.

In the 5 patients where no bypass graft was patent there was an increase in intercoronary patterns from 12 before operation to 16 afterwards. Five homocoronary and 2 mixed patterns remained unchanged.

There were 13 patients in whom one graft remained patent and one blocked. Preoperatively we demonstrated II homocoronary collateral systems, 17 intercoronary, and 2 mixed. Postoperatively 16 new intercoronary systems were shown in 12 patients: these were from the distal area of the bypassed artery to the peripheral branches of the artery with a non-patent graft (Fig.). In the remaining 4 new collateral systems were between other vessels not associated with the successfully bypassed artery. The original collateral vessels were

TABLE Sixteen patients with 2 grafts patent

\begin{tabular}{|c|c|c|}
\hline Types of collaterals & Preoperative & Postoperative \\
\hline Homocoronary & 8 & $\mathbf{I}$ \\
\hline Intercoronary & $2 I$ & I \\
\hline Mixed & 3 & $\mathbf{I}$ \\
\hline Total & 32 & 3 \\
\hline
\end{tabular}




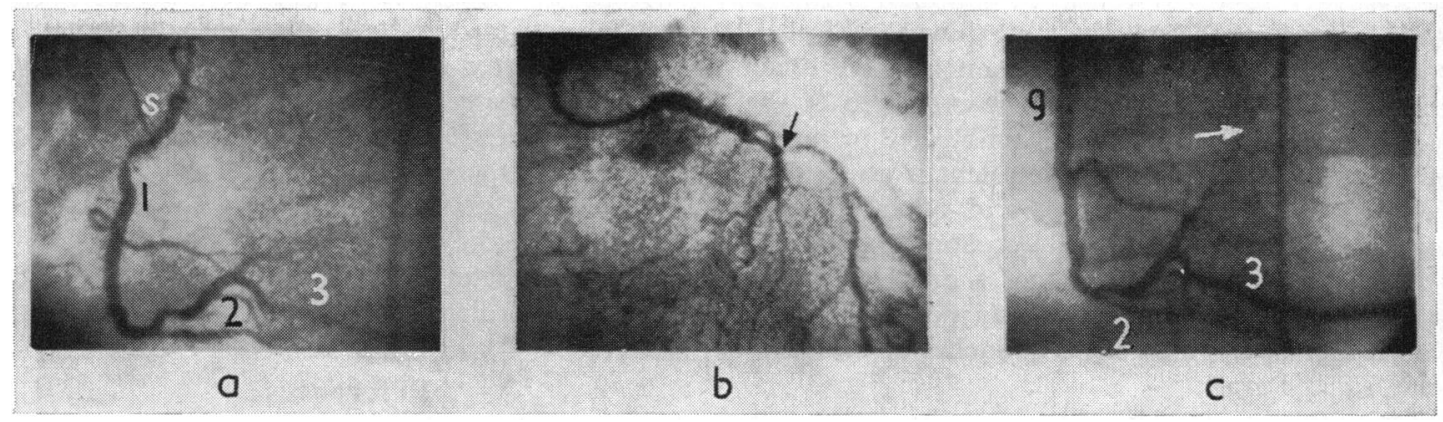

FIG. Example of the growth of a new collateral system after surgery in a patient with a patent graft to the right coronary artery. a) Right coronary artery injection in the left anterior oblique projection. The main stem shows a severe segmental stenosis distal to the origin of the sinus node artery; no collaterals are seen. b) Left coronary artery injection in the right anterior oblique projection. The left circumflex branch is stenosed (arrow) I to $2 \mathrm{~cm}$ from the origin. c) Postoperative, left anterior oblique projection. Selective injection of the graft in the right coronary artery. After grafting of the right coronary artery a new collateral channel is visualized connecting a small posterior left ventricular branch of the right coronary to the left circumflex (arrow). The left graft is occluded.

$I=$ main trunk of the right coronary artery; $2=$ acute marginal branch; $3=$ posterior descending artery; $s=$ sinus node artery.

homocoronary in II cases, and these could not be seen postoperatively in 5 patients with patent grafts. In 3 patients with blocked grafts the preoperative homocoronary collaterals were unchanged, and in a further 3 a new pattern had developed.

\section{Discussion}

Although a number of anatomical studies have shown that anastomoses of several hundred micrometres in diameter are present in normal human hearts (Vastesaeger et al., 1957; Bellman and Frank, 1958), selective coronary arteriography has never shown anatomical connexions forming anastomoses between normal coronary arteries (Gensini and $\mathrm{Da}$ Costa, 1969; Gensini and Esente, 1972). On the other hand, in large series of patients with severe occlusive coronary artery disease anastomotic channels can be well visualized (Gensini and $\mathrm{Da}$ Costa, 1969; Gensini and Esente, 1972; Helfant, Vokonas, and Gorlin, 1971).

It has been shown experimentally that during long-standing obstruction of a coronary artery in an otherwise normal animal heart, the flow through the anastomoses increases progressively, and that, whatever is the fundamental initiating factor, ligation of the artery represents a permanent stimulus to a progressive dilatation of the coronary anastomoses (Blumgart et al., 1950; Blum, Schauer, and Calef, 1938; Burchell, 1940). When chronic occlusive disease affects human coronary arteries a situation in some ways similar to that of the experimental coronary ligation is established and presumably the same haemodynamic process develops. Postmortem studies have shown that in states associated with chronic myocardial hypoxia, anastomoses of $\mathrm{I}$ to 2 $\mathrm{mm}$ in diameter are commonly found (James, 196I). In the vast majority of our patients, who had several years' history of angina, large and tortuous anastomoses were seen preoperatively. However, with one exception, in those patients in whom the inserted grafts were functioning well, all the coronary collateral pathways which were present before surgery could not be visualized at further study. These findings strongly suggest that once adequate anterograde flow is established in an occluded vessel to an area previously supplied by collateral anastomotic pathways, the blood-carrying function of the collateral vessels is no longer maintained. This does not necessarily imply anatomical changes in the anastomotic vessels, but their radiological disappearance suggests that they may revert to their primitive role of non-functioning vessels and presumably become 'functionally closed'. One explanation for this is that a significant pressure gradient between the coronary arteries represents the main factor for the functional opening of collateral channels, and that the radiological disappearance of collaterals between 2 bypassed arteries with well-functioning grafts implies the cancellation of that pressure gradient.

In some of the patients in whom collateral path- 
ways, visible preoperatively, have disappeared postoperatively, new collateral channels have been visualized at restudy. This can be explained by the fact that, when good flow is re-established by means of the graft, a new pressure gradient is established between the bypassed artery and other stenosed non-grafted arteries. A similar situation occurred in our patients in whom only I of 2 grafts remained patent; new collaterals commonly developed from the revascularized area to the branches of the artery with a failed graft.

Five out of 18 patients in whom the grafts were occluded developed myocardial infarction postoperatively in the territory of distribution of the grafted arteries. All these 5 infarcts occurred in the II patients who had shown some anterograde flow through the stenosis preoperatively. In each of the 5 patients with infarction the arteries showed postoperative loss of anterograde filling, but good collateral filling. This finding strongly suggests that a good collateral circulation in an ischaemic area of the myocardium does not necessarily protect against necrosis (Helfant et al., 1971). This finding is relevant to the rate of change in the collateral circulation. The collaterals rapidly vanish after successful revascularization and presumably have shut down, rather than occluded: this implies that the vessels could open up to provide an immediate blood flow to the area should a new occlusion of the coronary artery occur. However, when a stenosed coronary artery closes completely in relation to surgery and the bypass graft also blocks, there is no pre-existing collateral circulation available, and myocardial infarction is very likely.

\section{Conclusions}

Successful coronary artery bypass surgery leads to a disappearance of collateral vessels which previously supplied ischaemic areas. A patent functioning graft also allows the development of new collateral vessels from the grafted vessel to other stenosed non-grafted vessels or to other vessels receiving occluded grafts. If surgery is unsuccessful, i.e. if the inserted grafts (one or more) are occluded there is no change in the pattern of preoperative collateral vessels. If the graft is unsuccessful and there is further stenosis or a total block of the vessel at the site of graft insertion new collaterals may grow towards the grafted artery. The presence of these new collaterals does not necessarily protect against myocardial infarction in that area.

\section{References}

Bellman, S., and Frank, H. A. (1958). Intercoronary collaterals in normal hearts. Fournal of Thoracic Surgery, 36, 584.

Blum, L., Schauer, G., and Calef, B. (1938). Gradual occlusion of a coronary artery: an experimental study. American Heart fournal, 16, 159.

Blumgart, H. L., Zoll, P. M., Freedberg, A. S., and Gilligan, D. R. (1950). The experimental production of intercoronary arterial anastomoses and their functional significance. Circulation, 7, ro.

Burchell, H. B. (1940). Adjustments in coronary circulation after experimental coronary occlusion. Archives of Internal Medicine, 65, 240.

Gensini, G. G., and Da Costa, B. C. B. (1969). The coronary collateral circulation in living man. American fournal of Cardiology, 24, 393.

Gensini, G. G., and Esente, P. (1972). Anatomia radiologica della circolazione collaterale coronarica. Giornale Italiano di Cardiologia, 2, 604.

Helfant, R. H., Kemp, H. G., and Gorlin, R. (1970). Coronary atherosclerosis, coronary collaterals, and their relation to cardiac function. Annals of Internal Medicine, 73, 189.

Helfant, R. H., Vokonas, P. S., and Gorlin, R. (I97I). Functional importance of the human coronary collateral circulation. New England fournal of Medicine, 284, 1277.

James, T. N. (196I). Anatomy of the Coronary Arteries. Hoeber, New York.

Sheldon, W. C. (1969). On the significance of coronary collaterals. American fournal of Cardiology, 24, 303.

Vastesaeger, M. M., Van Der Straeten, P. P., Friart, J., Candaele, G., Ghys, A., and Bernard, R. M. (1957). Les anastomoses intercoronariennes telles qu'elles apparaissent à la coronarographie post mortem. Acta Cardiologica, 12, 365 .

Requests for reprints to Dr. P. R. Roy, Cardiac Department, National Heart Hospital, Westmoreland Street, London WIM 8BA. 